\title{
Estudio descriptivo del cribado del cáncer de cérvix en nuestro centro de salud. No captamos a la población de riesgo
}

\author{
Y. GonZález Rubio, M. S. CASTAÑo Pinto \\ Especialistas en Medicina Familiar y Comunitaria. \\ Centro de Salud Monóvar. Área 4 INSALUD. Madrid
}

\section{RESUMEN}

Fundamentos: la población inmigrante está aumentando en nuestro país y en nuestra zona de salud, y es una población con más riesgo para de sarrollar cáncer de cérvix que la población espa nola, así, nos planteamos la posibilidad de conocer cuál es la situación del cribado del cáncer de cér vix en esta población y compararla con la pobla ción local.

Objetivo: comparar el porcentaje de realización de citología vaginal entre la población inmigrante latinoamericana y la población local de un centro de salud.

Diseño: estudio observacional descriptivo transversal.

Material y métodos: en un centro de salud urba no, se estudió una muestra de 153 mujeres inmi grantes latinoamericanas y 153 mujeres españolas (muestra apareada por edad) de 18 a 65 años, de las que se recogieron datos sociodemográficos y de la asistencia al centro y datos sobre la realización de citología y frecuencia. Se analizaron los datos por comparación de porcentajes y Chi cuadrado.

Resultados: se encontraron diferencias signifi cativas entre ambos grupos en el porcentaje de mu jeres que se había realizado citología (25,5\% inmi grantes frente a 43,8\% no inmigrantes). También entre las que no se la habían realizado $(58,8$ frente a 41,2\%). En los distintos intervalos etarios sólo hubo diferencias en el de 30 a 39 años.

Conclusiones: dado que las mujeres inmigran tes presentan un porcentaje menor de realización
Descriptive study of cervix cancer screening in our Primary Health Care center: we do not accede the risk population

\section{ABSTRACT}

Backgrounds: since the immigrant population is increasing in our country and our zone of health, and has a higher incidence of cervix cancer, so, the possibility considers of knowing which is the situa tion of the screening of cancer of cervix in this population and comparing it with the local population.

Objective: to compare the percentage of accom plishment of vaginal cytology between the Latin American immigrant population and the local po pulation of a Primary Health Care center.

Design: cross-sectional descriptive observacio nal study.

Material and methods: in an urban center of health, we studied the sample of 153 Latin Ameri can women immigrants and 153 Spanish women (aged paired sample) of 18 to 65 years, on which socio-demographics data and on the attendance to the Primary Health Care center and the accom plishment of cytologies and frequency took shelter. The data by percent comparison and Chi Square test were analyzed.

Results: there were significant differences bet ween both groups in the percentage of women that had been made cytology (25.5\% immigrants as op posed to $43.8 \%$ non immigrants). Also between that they had not been made it $(58.8 \%$ as opposed to $41.2 \%)$. In the different aged intervals only there were differences in the one of 30 to 39 years.

Conclusions: since the immigrants women pre sent/display a smaller percentage of accomplish ment of cytology we would have to consider the 
de citologías deberíamos plantearnos la manera de mejorar dicha cobertura en esta población inmi grante, ya que al tener una incidencia más alta de cáncer de cérvix se puede beneficiar más del programa de cribado que la población española.

Palabras clave: Cáncer de cérvix. Citología va ginal. Mujeres latinoamericanas. Mujeres españo las. Cobertura. way to improve this cover in this immigrant population, since when having one more a higher incidence of cervix cancer can be benefited more from the sifting program that the Spanish popula tion.

Key words: Cervix cancer. Vaginal cytology. Latin American women. Spanish women. Cover.

\section{INTRODUCCIÓN}

La puesta en marcha de programas de actividades preventivas, requiere una gran inversión de recursos y por ello deben justificar su eficacia y eficiencia frente a otras alternativas de priorización. Las actividades preventivas pueden generar repercusiones negativas en los pacientes y efectos secundarios, como ansiedad innecesaria ante un posible diagnóstico, repetición de pruebas, uso de tratamientos de eficacia no comprobada, etc. La decisión de instaurar programas de prevención y promoción de la salud debe considerar factores en relación con el problema de salud, con la aplicación del programa y con las pruebas a realizar, y valorar la evidencia científica disponible sobre la recomendación de determinado programa ${ }^{1}$.

La evidencia científica existente sobre la eficacia de los programas de cribado de cáncer de cérvix proviene de los estudios de investigación; del análisis de las tendencias en registros sanitarios se ha observado una tendencia decreciente de las tasas de incidencia de cáncer invasivo en países donde el programa alcanzó gran cobertura poblacional ${ }^{2,3}$. Así, la efectividad y eficiencia de este programa están relacionadas con la frecuencia de presentación de este tumor y su historia natural, con la validez de la técnica de realización de la citología y sobre todo con la dificultad que supone la captación de pacientes de mayor riesgo ${ }^{4,5}$. En nuestro país, la citología exfoliativa tiene una sensibilidad que oscila entre el 74 y el $97,6 \%$ y una especificidad entre el 50 y el $91 \%{ }^{5,6}$. Si consideramos además que la baja frecuencia del tumor ocasiona un bajo valor predictivo positivo, el rendimiento del programa es pequeño.

España presenta una tasa de incidencia de cáncer de cérvix de 4-10 casos por 100.000 mujeres y Latinoamérica, zona de origen de gran parte de nuestra inmigración una tasa de 55 casos por $100.000^{2,7,8}$. Según diversos estudios ${ }^{9,10}$ hay sólo dos factores de riesgo diferentes entre ambas poblaciones, la edad de inicio de las relaciones y el número de parejas sexuales. La prevalencia del virus del papiloma humano, principal factor de riesgo para el cáncer de cérvix uterino es similar entre ambas poblaciones de mujeres ${ }^{11}$. La tasa de mortalidad en España es de 2,7 por 100.000 mujeres en $1994^{12}$ y en América del Sur oscila entre el 5-6 por 100.000 mujeres $^{3,12,13}$.

En la literatura revisada sobre la aplicación del programa en los distintos países no existe acuerdo en el inicio del cribado, la frecuencia de realización ni el rango etario que debe abarcar, pero la evidencia apunta a la realización de citología vaginal trianualmente, iniciándose a los 18 años ${ }^{4,14,15}$.

Los aspectos positivos que avalan la pertinencia de este programa son que la detección precoz del carcinoma in situ adecuadamente tratado asegura casi un $100 \%$ de curación, que haberse realizado una citología ya conlleva un beneficio individual a la mujer y que es un procedimiento bien aceptado por las pacientes ${ }^{4}$.

Al convivir en nuestro centro de salud mujeres españolas y mujeres inmigrantes latinoamericanas, nos planteamos un estudio cuyo objetivo fue valorar a cuántas mujeres llegó el programa de cribado, con qué periodicidad se realizaban la citología, si había diferencia en la aplicación del cribado en uno y otro grupo de población, y si había diferencias en los distintos grupos de edad. Hemos descrito las características demográficas y la cobertura sanitaria que presentan las pacientes latinoamericanas, y cuántas de estas mujeres han acudido al menos una vez al centro.

\section{MATERIAL Y MÉTODOS}

En nuestro centro de salud situado en un área urbana del noroeste de Madrid, se atiende 5.489 mujeres de edades comprendidas entre los 18 y los 65 años; de éstas un 2,9\% son inmigrantes latinoamericanas.

Hemos realizado un estudio observacional descriptivo transversal en el que se comparaba la cobertura del programa de realización de citología de cérvix uterino entre mujeres inmigrantes y mujeres no inmigrantes, que por el rango de edad deberían estar incluidas en dicho programa, pertenecientes a nuestro centro de salud. La fuente de datos fue la historia clínica. 
El tamaño muestral se seleccionó de acuerdo a un estudio piloto previo para unas proporciones esperadas de $\mathrm{p} 1=0,40$ y $\mathrm{p} 2=0,60$, con un error a de 0,05 y un potencia de 0,95 . Obteniéndose un tamaño muestral de 133 , que se aumentó hasta 153 por posibles pérdidas. Las mujeres fueron seleccionadas por un muestreo aleatorio estratificado por edad, en ambos grupos de mujeres (inmigrantes y no) de entre aquéllas que deberían estar incluidas en el programa de detección precoz del cáncer de cérvix, es decir, mujeres entre 18 y 65 años de edad.

Como primer paso, se estudiaron las características demográficas de estas mujeres dividiéndolas en cuatro grupos de edad (de 18 a 29, de 30 a 39, de 40 a 49 y de 50 a 65 años).

De las historias clínicas de las pacientes se extrajeron las variables del estudio tanto de tipo cuantitativo como la edad (en años), como de tipo cualitativo: tipo de cobertura sanitaria (pertenecientes al grupo de extranjeros sin recursos, dividido en dos categorías, sí o no), utilización de los servicios del centro de salud (es decir, si habían acudido al menos una vez al centro de salud, dividido también en las categorías sí o no), realización de citología de cérvix (dividido en cuatro categorías sí, no, acude a ginecología o acude al centro de orientación familiar), fecha de realización de la última citología (con tres intervalos: menos de tres años, de 3 a 5 años y más de 5 años), y adscripción al centro en los últimos dos años (dos categorías, una si las pacientes se han adscrito al centro durante los dos últimos años, y otra si lo han hecho anteriormente).

Se intentó el contacto telefónico con las pacientes que estaban incluidas en las categorías de utilización de servicios de ginecología y centro orientación familiar (COF) para averiguar si tenían o no realizada la citología, pero por la movilidad geográfica del grupo de mujeres inmigrantes fue prácticamente imposible contactar con la mayoría de las mujeres, por lo que hablamos posteriormente con el Servicio de Anatomía Patológica-Citología, que es el servicio que procesa las citologías realizadas por el ginecólogo de la zona, y con el COF, para obtener los datos referentes a estas mujeres.

Respecto al análisis estadístico primero se realizó un estudio descriptivo de la muestra de tal forma que para las variables cuantitativas se describirán la media y la desviación estándar; y para las variables cuantitativas la distribución porcentual de frecuencias y su error estándar. Posteriormente, se realizó una comparación de porcentajes y un análisis bivariante con la prueba Chi-cuadrado para variables cualitativas. En todo caso se definió un nivel de significación estadística del $5 \%$. Todos esos análisis se llevaron a cabo con el paquete estadístico SPSS versión 10.0 para Windows.

\section{RESULTADOS}

Los datos sociodemográficos obtenidos en el grupo de mujeres inmigrantes son los siguientes: la frecuencia de mujeres en cada grupo de edad fue, en el grupo de 18 a 29 años había 40 mujeres $(26,1 \%)$, de 30 a 39 años había 65 mujeres $(42,5 \%)$, de 40 a 49 años había 29 mujeres (19\%) y de 50 a 65 años había 19 mujeres $(12,4 \%)$.

Los datos de frecuencia en cada grupo de edad en las mujeres no inmigrantes eran idénticos debido a la forma de selección de la muestra (aleatorio estratificado por edad).

Del total de mujeres inmigrantes, 38 (24,8\%) estaban codificadas en tarjeta sanitaria como extranjeros sin recursos y $117(76,5 \%)$ se habían inscrito en el centro después del año 1998.

Del total de las mujeres inmigrantes, 115 $(75,2 \pm 6,8 \%)$ habían acudido alguna vez a la consulta en el centro de salud, y de éstas, 110 $(71,9 \pm 7,1 \%)$ desde el año 1998. Del total de las mujeres no inmigrantes $135(88,2 \pm 5,1 \%)$ habían acudido alguna vez a la consulta, y de éstas, 94 $(61,4 \pm 7,7 \%)$ desde el 1998 hasta la actualidad (Fig. 1). Al comparar los porcentajes de mujeres que consultaron al menos una vez existían diferencias significativas $(\mathrm{p}<0,05)$ entre las inmigrantes y las no inmigrantes $(75,2 \pm 6,8 \%$ frente a $88,2 \pm 5,1 \%)$.

Se obtuvieron los siguientes datos de la realización de citología:

-Entre las mujeres inmigrantes, $39(25,5 \pm 6,9 \%)$ se habían realizado al menos una citología, $90(58,8 \pm 7,7 \%)$

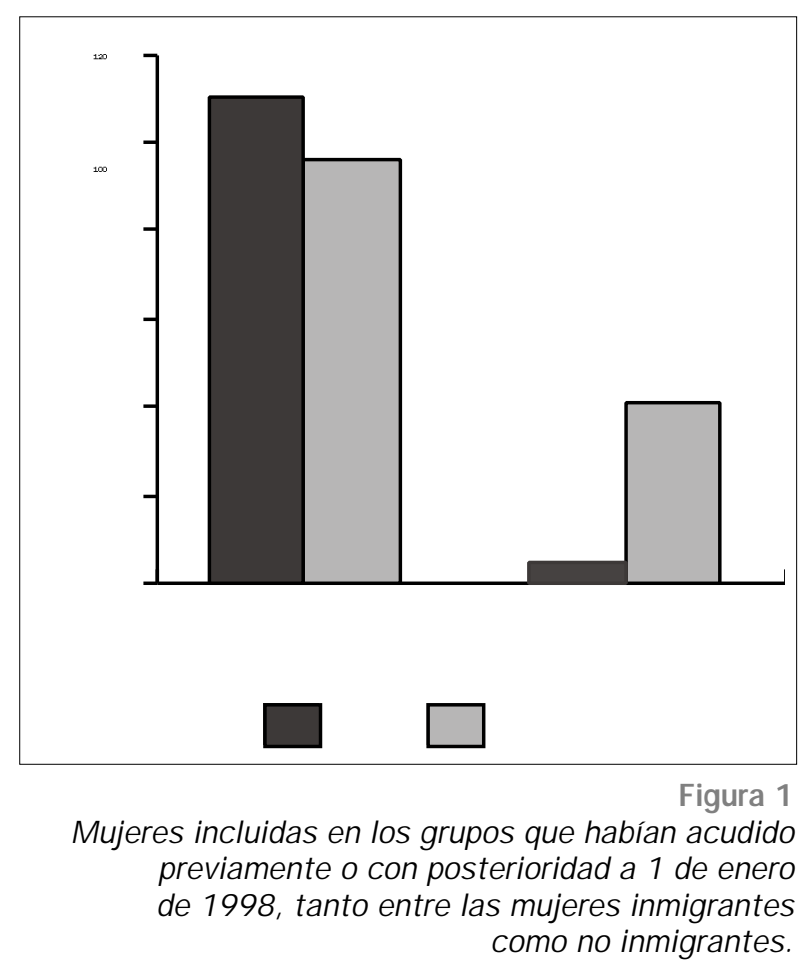


no tenían registrada ninguna, $14(9,2 \pm 4,5 \%)$ acudían a control por el ginecólogo en atención especializada y $10(6,5 \pm 3,9 \%)$ acudían a control en el COF (Fig. 2). Si añadimos al total de las citologías realizadas el número de mujeres que se la hicieron en el ginecólogo o en el COF, ya que no todas las mujeres incluidas en estos dos grupos tenían realizada la citología, obtenemos un total de 49 mujeres que se habían realizado al menos una citología $(32 \pm 7,3 \%)$ y 104 sería el total de mujeres que no la tienen registrada, ni la hicieron en el ginecólogo, ni en el COF (68 $\pm 7,3 \%)$ (Fig. 3).

-Entre las mujeres no inmigrantes 67 $(43,8 \pm 7,8 \%)$ se habían realizado al menos una citología, $63(41,2 \pm 7,8 \%)$ no tenía registrada ninguna en la historia clínica, $18(11,8 \pm 5,1 \%)$ seguían controles por el ginecólogo de atención especializada y $5(3,3 \pm 2,8 \%)$ acudían a los servicios de planificación sanitaria. (Fig. 2). Si añadimos igualmente las mujeres incluidas en los grupo de atención en el ginecólogo y en el centro de orientación familiar, obtenemos un total de 78 mujeres no inmigrantes que se habían realizado al menos una citología $(51 \pm 7,9 \%)$ y de 75 mujeres que no tenían citología registrada $(49 \pm 7,9 \%)$ (Fig. 3).

-Respecto al intervalo de realización de la citología en el grupo de mujeres latinoamericanas, 31 $(81,6 \pm 6,1 \%)$ se habían realizado la última hacía menos de tres años, $2(5,3 \pm 3,5 \%)$ entre los últimos tres a cinco años, $5(13,2 \pm 5,3 \%)$ hacía más de cinco años, y en 1 caso la fecha de realización no estaba

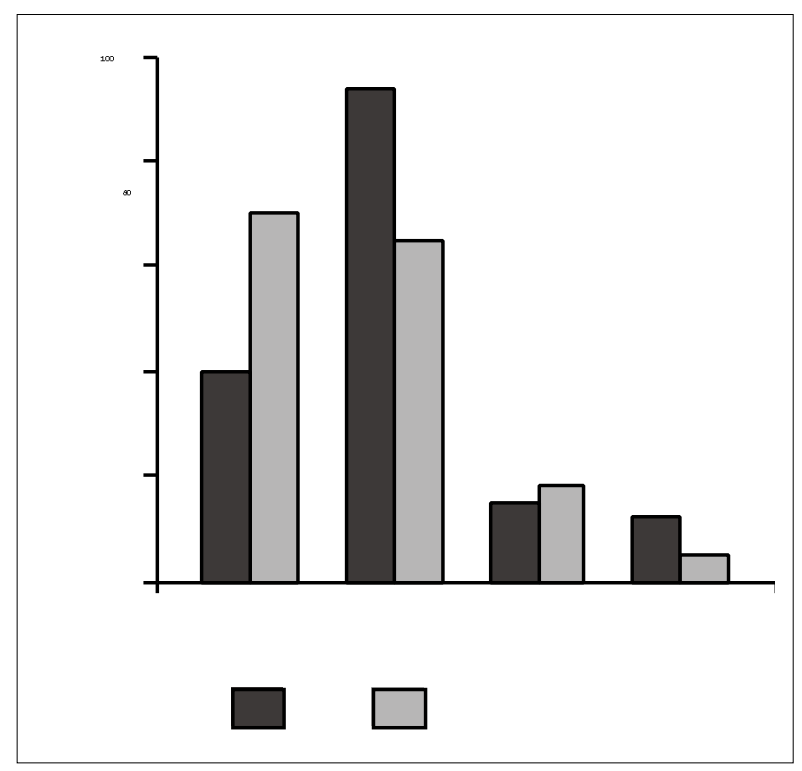

Figura 2

Mujeres incluidas en los distintos grupos de citología realizada (sí), no registrada (no), acudieron a ginecología (ginecólogo) o acudieron al centro de orientación familiar (COF) según pertenezcan al grupo de inmigrantes o no inmigrantes.

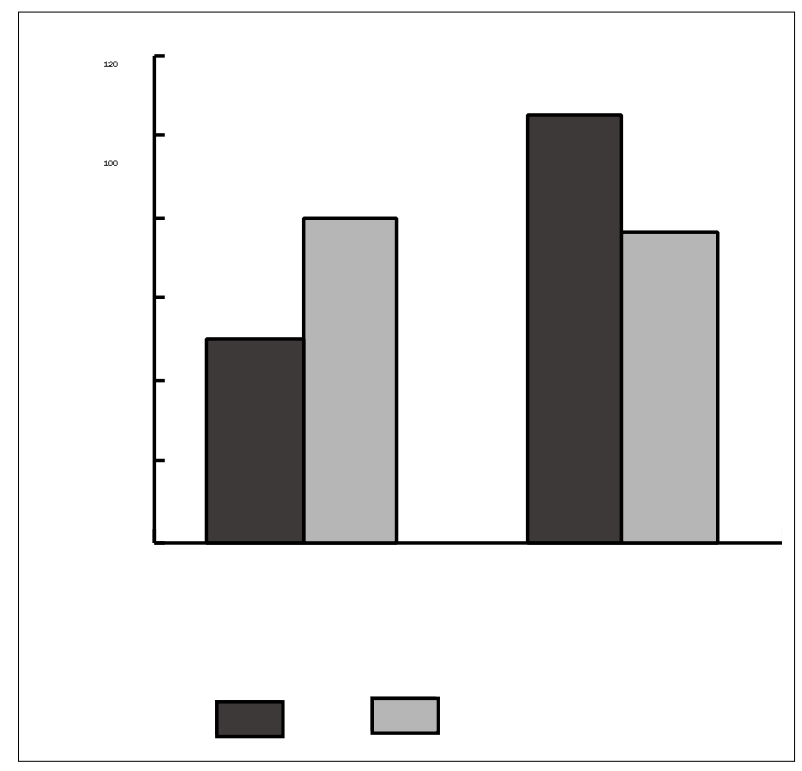

Figura 3

Mujeres incluidas en los distintos grupos de realización o no de citología (agrupando las que van a ginecólogo y al COF con las que se realizan citología o no citología) según pertenezcan al grupo de inmigrantes o no inmigrantes.

registrada en la historia clínica. Y entre las mujeres no inmigrantes $31(44,9 \pm 7,5 \%)$ se habían realizado la última hacía menos de tres años, $19(27,5 \pm 7,0 \%)$

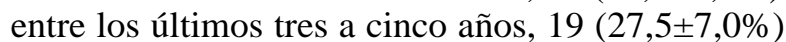
hacía más de cinco años.

Considerando los porcentajes de realización de citología y las diferencias entre ambos grupos se obtuvieron los siguientes resultados: se encontraron diferencias significativas $(\mathrm{p}<0,05)$ entre el total de mujeres inmigrantes que se habían realizado citología y las no inmigrantes $(25,5 \pm 6,9$ frente a $43,8 \pm 7,8 \%$ ), también entre las que no se la había realizado $(58,8 \pm 7,7$ frente a $41,2 \pm 7,8 \%)$, pero no se encontraron diferencias significativas entre las que acudían al ginecólogo ni entre las que acudían al COF. Sólo se obtuvieron diferencias significativas entre las mujeres del intervalo etario de 30 a 39 años y no en el resto de intervalos (Tablas I y II).

\section{DISCUSIÓN}

En los últimos años está habiendo una explosión demográfica de población inmigrante en nuestro país y en nuestra zona de salud esa inmigración es mayoritariamente de Latinoamérica. Es una población que por sus características socio-económicas parece tener gran movilidad de residencia, como se puede apreciar en el dato de que el $76,5 \%$ de las mujeres inmigrantes de nuestro centro de salud se hayan inscrito en éste en los últimos tres años. 
Tabla I

PORCENTAJES EN LAS DISTINTAS CATEGORIAS DE REALIZACIÓN O NO DE CITOLOGÍAS PARA LAS MUJERES INMIGRANTES O NO INMIGRANTES; INCLUYE LOS VALORES ABSOLUTOS Y EL INTERVALO DE CONFIANZA PARA UNA $p<0,05$

\begin{tabular}{lccc}
\hline № de pacientes (\%) & Inmigrantes & No inmigrantes & p de la diferencia \\
\hline Al menos una citología & $39(25,5 \pm 6,9 \%)$ & $67(43,8 \pm 7,8 \%)$ & $<0,05$ \\
Ninguna citología & $90(58,8 \pm 7,7 \%)$ & $63(41,2 \pm 7,8 \%)$ & $<0,05$ \\
Control ginecólogo & $14(9,2 \pm 4,5 \%)$ & $18(11,8 \pm 5,1 \%)$ & No significativa \\
Control planificación familiar & $10(6,5 \pm 3,9 \%)$ & $5(3,3 \pm 2,8 \%)$ & No significativa \\
\hline Total & 153 & 153 & \\
\hline
\end{tabular}

\section{Tabla II}

PORCENTAJES EN LAS DISTINTAS CATEGORIAS DE REALIZACION O NO DE CITOLOGIAS PARA LAS MUJERES INMIGRANTES O NO INMIGRANTES, SEGUN LOS DISTINTOS INTERVALOS DE EDAD; INCLUYE LOS VALORES ABSOLUTOS Y EL INTERVALO DE CONFIANZA PARA UNA $p<0,05$

\begin{tabular}{lccc}
\hline № de pacientes (\%) & Inmigrantes & No inmigrantes & p de la diferencia \\
\hline De 18 a 29 años & $40(26,1 \pm 6,9 \%)$ & $40(26,1 \pm 6,9 \%)$ & \\
Al menos una citología & $3(7,5 \pm 4,1 \%)$ & $9(22,5 \pm 6,6 \%)$ & No significativa \\
Ninguna citología & $30(75 \pm 6,8 \%)$ & $24(60,0 \pm 7,7 \%)$ & No significativa \\
Control ginecólogo & $2(5 \pm 3,4 \%)$ & $5(12,5 \pm 5,2 \%)$ & No significativa \\
Control planif. fam. & $5(12,5 \pm 5,2 \%)$ & $2(5,0 \pm 3,4 \%)$ & No significativa \\
& & & \\
De 30 a 39 años & $65(42,5 \pm 7,8 \%)$ & $65(42,5 \pm 7,8 \%)$ & $<0,05$ \\
Al menos una citología & $20(30,8 \pm 7,3 \%)$ & $35(53,8 \pm 7,9 \%)$ & $<0,05$ \\
Ninguna citología & $34(52,3 \pm 7,9 \%)$ & $21(32,3 \pm 7,3 \%)$ & No significativa \\
Control ginecólogo & $7(10,8 \pm 4,9 \%)$ & $7(10,8 \pm 4,9 \%)$ & No significativa \\
Control planif. fam. & $4(6,2 \pm 3,8 \%)$ & $2(3,1 \pm 2,7 \%)$ & No significativa \\
& $29(19,0 \pm 6,2 \%)$ & $29(19,0 \pm 6,2 \%)$ & No significativa \\
De 40 a 49 años & $7(24,1 \pm 6,7 \%)$ & $10(34,5 \pm 7,3 \%)$ & No significativa \\
Al menos una citología & $17(58,6 \pm 7,7 \%)$ & $14(48,3 \pm 7,7 \%)$ & \\
Ninguna citología & $4(13,8 \pm 5,1 \%)$ & $4(13,8 \pm 5,1 \%)$ & No significativa \\
Control ginecólogo & $1(3,4 \pm 2,7 \%)$ & $1(3,4 \pm 2,7 \%)$ & No significativa \\
Control planif. fam. & & & No significativa \\
& $19(12,4 \pm 5,2 \%)$ & $19(12,4 \pm 5,2 \%)$ & - \\
De 50 a 65 años & $9(47,4 \pm 7,9 \%)$ & $13(68,4 \pm 7,3 \%)$ & \\
Al menos una citología & $9(47,4 \pm 7,9 \%)$ & $4(21,1 \pm 6,2 \%)$ & \\
Ninguna citología & $1(5,3 \pm 3,4 \%)$ & $0(10,5 \pm 5,1 \%)$ & $0-$ \\
Control ginecólogo & $0-$ & 153 & \\
Control planif. fam. & 153 & 153 & \\
\hline Total & & &
\end{tabular}

A pesar de la sensación de hiperfrecuentación de esta población y de que gran parte de ésta se debe a la petición aparentemente excesiva de citologías vaginales, si nos fijamos en el porcentaje de mujeres que han acudido en ambos grupos y en la cobertura del programa de citologías en nuestro centro nos encontramos con la sorpresa de que estos datos son contradictorios con nuestra impresión inicial.

El porcentaje de mujeres inmigrantes que no han acudido nunca al centro es mayor que el de las no inmigrantes; pero, considerando sólo el periodo de los tres últimos años, las mujeres inmigrantes han acudido con más frecuencia; han acudido un 71,9 frente al $61,4 \%$. Se considera que al cabo de 2 años, más del 95\% de la población ha acudido al menos una vez a su médico ${ }^{16}$. Las mujeres latinoamericanas habrían acudido al médico, como cualquier otro grupo de población.

Respecto a la realización de la citología, el porcentaje de mujeres que no tienen ninguna citología registrada en la historia clínica es mayor entre las 
inmigrantes que entre las no inmigrantes. A pesar de haber acudido durante los últimos tres años con más frecuencia al centro de salud, no se les ofertó el programa de cribado, aún conociendo que estas mujeres constituyen una población de mayor riesgo que las españolas.

Cuando se analizaron las diferencias respecto a la realización de citología en los distintos intervalos de edad sólo se encontraron diferencias significativas en el intervalo de 30-39 años, intervalo en el que estaban incluidas más mujeres. Este dato probablemente se explica por el pequeño número de mujeres incluidas en los otros intervalos, y pensamos que en un estudio con mayor número de mujeres se encontrarían las mismas diferencias que en el intervalo de 30 a 39 años y que en el total de mujeres.

Revisando la bibliografía es difícil encontrar estudios similares referentes a programas de cribado de cáncer de cérvix entre poblaciones diferentes. Sí se encuentran otros estudios que analizan la frecuentación y causas de la frecuentación en población inmigrante ${ }^{17,18}$. Y hemos encontrado artículos en los que se analizan de forma separada distintos factores de riesgo para cáncer de cérvix (entre ellos la realización o no de cribado) realizados entre población española, residente en España y población colombiana, residente en Colombia ${ }^{9}$ y en los resultados obtenidos se encuentran diferencias con los resultados de nuestro estudio (ellos no encuentran diferencias significativas entre las dos poblaciones en la realización de cribado). Estos estudios con cada una de las poblaciones en su respectivo entorno no nos parecen comparables con estudios en poblaciones inmigrantes en un país que no es el propio (incluso con problemas con la legalidad de su estancia en ese país).

Una de las dificultades encontradas en la realización de este estudio en el centro de salud fue la necesidad de combinar varios registros lo que en algún momento puede haber llevado a perder alguna paciente. Y pese a la falta de un registro informático accesible para conseguir la población inmigrante el porcentaje de población inmigrante obtenido $(2,9 \%)$ se corresponde con lo esperable según datos del Ministerio del Interior ${ }^{19}$.

Las posibles causas de encontrar tan baja cobertura en el programa de prevención de cáncer de cérvix en las mujeres inmigrantes (pero también en las no inmigrantes), pueden ser varias: por un lado la falta de registro de esta actividad en la historia clínica, puede haberse realizado la citología pero no haber sido recogida, lo que desde el punto de vista de este estudio que basaba la recogida de datos en la historia clínica, ha sido imposible de subsanar. Por otro lado habría que tener en cuenta que las mujeres que no han acudido nunca a la consulta pudieran estar siguiendo controles gine- cológicos o en el servicio de orientación familiar, de forma privada o en otras instituciones (por ejemplo Ayuntamiento) y quedaría registrado como no realizada en la recogida de datos; y este hecho tampoco es posible subsanarlo a no ser con una recogida de datos directamente de las pacientes. Aunque nos ha sorprendido conocer el dato de que más de la mitad de las mujeres que solicitaron en nuestras consultas una derivación para el ginecólogo o el COF, no tenían realizada la citología, y el motivo principal fue que ni siquiera acudieron a dicha cita concertada.

Considerando que la incidencia del cáncer de cérvix en la población latinoamericana es una de las más elevadas, 55 casos por cada 100.000 mujeres, (aproximadamente diez veces más que en la población española) y los datos de cobertura del programa de cribado obtenidos en nuestro estudio deberíamos plantearnos la manera de mejorar dicha cobertura en esta población inmigrante, ya que al tener una incidencia tan alta se puede beneficiar más del programa de cribado que la población española. Una de las formas de aumentar la cobertura es seguir las recomendaciones del PAPPS $^{2}$ de búsqueda activa de las mujeres con más factores de riesgo, y es que estas mujeres latinoamericanas, por su mayor incidencia de cáncer de cérvix serían ya mujeres de riesgo. Otra forma sería fomentar la participación activa de la población en las campañas de cribado con información a la población susceptible de beneficiarse de ellas desde la Atención Primaria. Así pues, búsqueda activa, información a la población y confirmación de haberse hecho la citología, nos parece primordial.

No podemos terminar este artículo sin recordar que ha sido realizado sólo con la población de mujeres inmigrantes latinoamericanas de un centro de salud y que probablemente sería necesario realizar estudios más amplios para conseguir resultados más concluyentes. Nuestra impresión es que no se es riguroso en la aplicación del programa y que no estamos llegando a las mujeres de más riesgo, y por el contrario desde ciertas sociedades (ginecólogos privados o no) aconsejan y realizan anualmente el cribado a las mujeres que les visitan (en general mujeres de mayor nivel socioeconómico, más motivadas y en principio de menor riesgo). Por eso sugerimos que podrían realizarse nuevos estudios sobre si la implantación de medidas como las anteriormente descritas para la captación de mujeres latinoamericanas y otras mujeres de riesgo de cáncer de cérvix en las campañas de cribado aumentaría o no la cobertura de dichas campañas, y estudios sobre si esto tiene algún efecto en el estado de salud de nuestras pacientes, es decir, si realmente se disminuye la incidencia del cáncer de cérvix. 


\section{AGRADECIMIENTOS}

Nuestro agradecimiento a $\mathrm{D}^{\mathrm{a}}$ Teresa Domínguez, auxiliar de enfermería del Centro de Salud Monóvar, al Dr. González Palacios, jefe de servicio de Anatomía Patológica del Hospital Ramón y Cajal y a su servicio, y al Centro de Orientación Familiar, por la colaboración que nos han prestado.

\section{CORRESPONDENCIA:}

María Soledad Castaño Pinto

Centro de Salud Monóvar

C/ Monóvar, 11

28033 Madrid

\section{Bibliografía}

1. Martín Zurro A, Gené Badia J, Subías Loren P. Actividades preventivas y de promoción de la salud. En: Martín Zurro A, Cano Pérez J, eds. Atención Primaria. Conceptos, organización y práctica clínica. Barcelona: Harcourt SA, 1999. p. 421-37.

2. Sigurdson K, Adalsteinsson S, Ragnarsson J. Trends in cervical and breast cancer in Iceland. A statiscal evauation of trends in incidence and mortality for the period 1955-1989. The relation to screening and prediction to the year 2000. Int J Cancer 1991; 48: 523-8.

3. Quinn M, Babb P, Jones J, Allen E. United Kingdom Association of Cancer Registries. Effects of screening on incidence of mortality from cancer in England: evaluation based on routinely collected statics. Br Med J 1999; 318: 904-8.

4. Alonso JM, Bellas B, Cierco P, Gálvez I, González J, Martín N, et al. Prevención del cáncer. Aten Primaria 1999; 24 (Supl. 1): 83-6.

5. Cuirana Misol R, Brotons Cuixart C, Forés García MD, Actividades de prevención y promoción de la salud en el adulto. En: Martín Zurro A, Cano Pérez J, eds. Atención Primaria. Conceptos, organización y practica clínica. Barcelona: Harcourt SA, 1999. p. 469-504.

6. Borras JM, Iglesias X. Cribado de cáncer de cuello de útero. Med Clin (Barc) 1994; 102 (Supl. 1): 80-4.

7. Parkin DM, Whelan SL, Ferlay J, Raymond L, Young J (eds). Cancer incidence in Five Continents (Vol VII). IARC Scientific. Publication no 143. Lyon, 1997.

8. Gustafsson L, Pontén J, Bergström R, Adam H-O. Internacional incidence rates of invasive cervical cancer before cytological screning. Int J Cancer 1997; 71; 159-65.

9. Bosch FX, Muñoz N, De San José S, Izarzugaza I, Gili M, Viladiu P, et al. Risk Factors for Cervical Cancer in Colombia and Spain. Int J Cancer 1992; 52: 750-8.

10. De San José S, Muñoz N, Bosch FX, Reimann K, Pedersen NS, Orfilia J, et al. Sexually transmited agents and cervical neoplasia in Colombia and Spain. Int J Cancer 1994; 56: 358-63.

11. Bosch FX, Manos MM, Muñoz N, Sherman M, Jansen AJ, Schifffman MH, et al. Prevalence of Human Papillomavirus in Cervical Cancer: a Worldwide Perspective. Natl Cancer Inst 1995; 87: 796-802.

12. Rivas A, Salcedo A. Cáncer ginecológico. En: Martín Zurro A, Cano Pérez JF, eds. Atención Primaria. Conceptos, organización y practica clínica. Barcelona: Doyma SA, 1994. p. 910-24.

13. Restrepo H, González J, Roberts E, Litvak J. Epidemiología y control del cáncer de cuello uterino en America Latina y Caribe. Bol of Sanit Panam 1987; 102 (6): 578-92.

14. Herrero H, Brinton LA, Reeves WC, Brenes MM, De Britton RC, Gaitan E, et al. Screening for cervical cancer in Latin América: a case-control study. Int J Epidemiol 1992; 21: 1050-6.

15. Day NE. Screening for cáncer de cérvix. J Epidemiol Community Health 1989; 43: 103-6.

16. Gené Badia J, Jiménez Villa J, Martín Sánchez A. Historia Clínica, sistemas de registro e información. En: Martín Zurro A, Cano Pérez JF, eds. Atención Primaria. Conceptos, organización y práctica clínica. Barcelona: Doyma SA, 1994. p. 141-57.

17. Sanz B, Torres MA, Schumacher R. Características sociodemográficas y utilización de servicios sanitarios por la población inmigrante residente en un área de la Comunidad de Madrid. Aten Primaria 2000; 26: 314-8.

18. Lacalle Rodríguez-Labajo M, Gil Juberías G, Sagardui Villamor JK, González López E, Martínez Ruiz R, Orden Martínez B. Resultados de la aplicación de un examen de salud en la población inmigrante. Aten Primaria 2000; 25: 634-8.

19. Ministerio del interior. En documentos semFYC, $\mathrm{n}^{\circ} 17$. La atención al inmigrante, del aluvión a la solución razonable. Diciembre 2000 